$$
\text { "vincze" — 2009/5/28 - 21:20 — page } 87 \text { — \#1 }
$$

\title{
On a special class of generalized conics with infinitely many focal points
}

\author{
Ábris Nagy, Zsolt RÁBAi and Csaba Vincze
}

\begin{abstract}
Let a continuous, piecewise smooth curve in the Euclidean space be given. We are going to investigate the surfaces formed by the vertices of generalized cones with such a curve as the common directrix and the same area. The basic geometric idea in the background is when the curve runs through the sides of a non-void triangle $A B C$. Then the sum of the areas of some triangles is constant for any point of such a surface. By the help of a growth condition we prove that these are convex compact surfaces in the space provided that the points $A, B$ and $C$ are not collinear. The next step is to introduce the general concept of awnings spanned by a curve. As an important example awnings spanned by a circle will be considered. Estimations for the volume of the convex hull will be also given.
\end{abstract}

Key words and phrases: convexity, conics.

ZDM Subject Classification: Primary: 53A04; Secondary: 52A10, 52A27.

\section{Introduction}

The concept of generalized conics as the set of points $\vec{x} \in \mathbb{R}^{n}$ such that

$$
\int_{G} \alpha(\vec{g}) d(\vec{g}, \vec{x}) d g \leq \rho
$$

can be found in [2], p. 742. Here $G \subset \mathbb{R}^{n}$ is the set of foci, $\alpha: G \rightarrow \mathbb{R}$ is a weight function and $\rho$ is a real constant. As an open question the authors posed the Supported by OTKA F 049212, Hungary.

Copyright (c) 2009 by University of Debrecen 


$$
\text { "vincze" — 2009/5/28 - 21:20 — page } 88 \text { — \#2 }
$$

problem whether which results (of the classical case) can be extended to the cases of infinitely many focal points or to continuous set of foci. We are going to deal with a similar problem based on special choices of $G$ and a more general weight function admitting the points of the space in its argument. Our geometric idea can be formulated as follows: let three (or more, but finite many) points $A, B$ and $C$ be given in the three-dimensional euclidean space and consider the set of the points $P$ such that

$$
\mathcal{A}(A B P)+\mathcal{A}(A P C)+\mathcal{A}(P B C)=\text { const. }
$$

where $\mathcal{A}(A B P), \mathcal{A}(A P C)$ and $\mathcal{A}(P B C)$ denote the area of the triangles $A B P$, $A P C$ and $P B C$, respectively. We will show that (1) defines convex compact surfaces in the space provided that the points $A, B$ and $C$ are not collinear. In case of collinear points we have unbounded cylindrical surfaces. The global minimum of the left hand side as the function of $P$ is attained at any point contained by the convex hull of the triangle $A B C$. The next step is the substitution of the sides of the triangle $A B C$ with a continuous, piecewise smooth curve. We are going to investigate the niveau surfaces of the function $P \rightarrow \mathcal{A}(P)$ measuring the area of generalized cones with such a curve as the common directrix and the vertex $P$. They are called awnings spanned by the curve. As an important special case awnings spanned by a circle will be considered. We give estimations for the volume of the convex hull.

\section{The basic geometric idea}

Let the points $A, B$ and $C$ be given in the three-dimensional Euclidean space and consider the function

$$
\begin{aligned}
\Sigma(P):= & \frac{1}{2}|(P-A) \times(P-B)|+\frac{1}{2}|(P-C) \times(P-A)| \\
& +\frac{1}{2}|(P-B) \times(P-C)|
\end{aligned}
$$

measuring the sum of the areas of the triangles $A B P, A P C$ and $P B C$.

LEMMA $1 . \Sigma$ is a convex function.

ProOF. It is enough to prove the convexity of the expression

$$
|(P-A) \times(P-B)|=|P \times(A-B)+A \times B|
$$




$$
\text { "vincze" — 2009/5/28 - 21:20 — page } 89-\# 3
$$

as the function of $P$. Here

$$
f(P):=P \times(A-B)+A \times B
$$

is an affine mapping preserving the affine, especially the convex combination of the points, i.e.

$$
f(\lambda P+(1-\lambda) Q)=\lambda f(P)+(1-\lambda) f(Q) .
$$

The triangle inequality implies that $\Sigma$ is convex.

Lemma 2. If $A, B$ and $C$ are not collinear then the global minimum is attained at the points of the convex hull of the triangle $A B C$.

Proof. According to the triangle inequality it can be easily seen that $\Sigma$ is great than or equal to the norm of the sum

$$
\frac{1}{2}(P-A) \times(P-B)+\frac{1}{2}(P-C) \times(P-A)+\frac{1}{2}(P-B) \times(P-C)
$$

which is just the area of the triangle $A B C$. If $P$ is not in the plane of $A B C$ then the orthogonal projection decreses the sum of the areas. If $P$ is not in the convex hull of the triangle $A B C$ then $P$ and, for example, the point $C$ are separated by the line $A B$. According to the Plane separation postulate the segment $P C$ meets the line $A B$ which implies that $A B C$ is covered by the union of the triangles $A B P, A P C$ and $P B C$.

LEMMA 3. If a function satisfies the growth condition

$$
\lim _{|P| \rightarrow \infty} \frac{\Sigma(P)}{|P|}:=\lim _{r \rightarrow \infty} \inf \left\{\frac{\Sigma(P)}{|P|}|| P \mid>r\right\}>0
$$

then it has bounded level sets.

Proof. Suppose, in contrary, that the level set

$$
M:=\{P \mid \Sigma(P) \leq c\}
$$

is unbounded. Then we can choose a sequence $P_{n}$ of the elements of $M$ such that the norm $\left|P_{n}\right|$ tends to the infinity. Therefore

$$
\lim _{|P| \rightarrow \infty} \frac{\Sigma(P)}{|P|} \leq \lim _{n \rightarrow \infty} \frac{\Sigma\left(P_{n}\right)}{\left|P_{n}\right|} \leq \lim _{n \rightarrow \infty} \frac{c}{\left|P_{n}\right|}=0,
$$

which is a contradiction. 


$$
\text { "vincze" — 2009/5/28 - 21:20 — page } 90 \text { — \#4 }
$$

REMARK 1. Requiring a function to have bounded level sets is also a kind of growth conditions. For convex functions these conditions are actually equivalent to each other as it was stated in [1] but the proof is left as an exercise.

Theorem 1. If $A, B$ and $C$ are not collinear then the levels of the function $\Sigma$ are convex and compact.

Proof. Since $\Sigma$ is continuous the levels are closed subset of the space. In order to see the compactness we prove that it satisfies the growth condition

$$
\lim _{|P| \rightarrow \infty} \frac{\Sigma(P)}{|P|}:=\lim _{r \rightarrow \infty} \inf \left\{\frac{\Sigma(P)}{|P|}|| P \mid>r\right\}>0
$$

which implies that the levels are bounded. First of all note that

$$
\begin{gathered}
\frac{1}{4}|(P-A) \times(P-B)|^{2}+\frac{1}{4}|(P-C) \times(P-A)|^{2} \\
+\frac{1}{4}|(P-B) \times(P-C)|^{2} \leq \Sigma^{2}(P) .
\end{gathered}
$$

The left hand side can be written into the form

$$
L_{2}(P)+L_{1}(P)+L_{0}
$$

with a quadratic form, a linear functional and a real constant denoted by $L_{2}, L_{1}$ and $L_{0}$, respectively. The steps of the proof can be summarized as follows:

- We prove that $L_{2}$ is positive definite.

- Taking $L_{1}(P)=\langle L, P\rangle$ we have that

$$
-|L| \leq \frac{L_{1}(P)}{|P|} \leq|L| .
$$

- If

then

$$
k:=\min \left\{\frac{L_{2}(P)}{|P|^{2}}|| P \mid \neq 0\right\}>0
$$

$$
\frac{\Sigma^{2}(P)}{r^{2}} \geq k-\frac{|L|}{r}+\frac{L_{0}}{r^{2}}, \quad \text { where } r=|P| .
$$

Taking the limit $r \rightarrow \infty$ it follows that

$$
\lim _{|P| \rightarrow \infty} \frac{\Sigma(P)}{|P|} \geq \sqrt{k}>0
$$

as was to be stated. 
Since

$$
(P-A) \times(P-B)=P \times(A-B)+A \times B=P \times A-P \times B+A \times B
$$

we have that

$$
\begin{aligned}
\mid(P-A) & \times\left.(P-B)\right|^{2}=\langle P \times A, P \times A\rangle+\langle P \times B, P \times B\rangle+\langle A \times B, A \times B\rangle \\
& -2\langle P \times A, P \times B\rangle+2\langle P \times A, A \times B\rangle-2\langle P \times B, A \times B\rangle .
\end{aligned}
$$

Using the identity

$$
\langle X \times Y, Z \times W\rangle=\langle X, Z\rangle\langle Y, W\rangle-\langle Y, Z\rangle\langle X, W\rangle
$$

to separate the quadratic term of the sum, it follows that

$$
\begin{gathered}
|(P-A) \times(P-B)|^{2}=|A|^{2}|P|^{2}-\langle A, P\rangle^{2}+2\langle A, P\rangle\langle B, P\rangle \\
-2|P|^{2}\langle A, B\rangle+|P|^{2}|B|^{2}-\langle P, B\rangle^{2}+\text { linear }+ \text { const. }
\end{gathered}
$$

and, consequently,

$$
\begin{gathered}
|(P-A) \times(P-B)|^{2}=|P|^{2}\left(|A|^{2}-2\langle A, B\rangle+|B|^{2}\right) \\
+(\langle A, P\rangle-\langle B, P\rangle)^{2}+\text { linear }+ \text { const. }=
\end{gathered}
$$

Therefore

$$
|(P-A) \times(P-B)|^{2}=|P|^{2}|A-B|^{2}-\langle P, A-B\rangle^{2}+\text { linear }+ \text { const. }
$$

and the quadratic form $L_{2}$ can be expressed as

$$
\begin{aligned}
L_{2}(P)= & \frac{1}{4}\left(|P|^{2}|A-B|^{2}-\langle P, A-B\rangle^{2}\right)+\frac{1}{4}\left(|P|^{2}|C-A|^{2}-\langle P, C-A\rangle^{2}\right) \\
& +\frac{1}{4}\left(|P|^{2}|B-C|^{2}-\langle P, B-C\rangle^{2}\right) .
\end{aligned}
$$

The Cauchy-Schwarz inequality shows that $L_{2}(P)$ vanishes if and only if $P$ is linear dependent with each of the vectors $A-B, C-A$ and $B-C$. Therefore $P$ is equal to zero or the points $A, B$ and $C$ are collinear.

REMARK 2. The linear term can be expressed as

$$
\begin{aligned}
L_{1}(P)= & \frac{1}{2}\langle P, A\rangle\left(\langle A, B+C\rangle-|B|^{2}-|C|^{2}\right)+\frac{1}{2}\langle P, B\rangle\left(\langle B, A+C\rangle-|A|^{2}-|C|\right) \\
& +\frac{1}{2}\langle P, C\rangle\left(\langle C, A+B\rangle-|A|^{2}-|B|^{2}\right)
\end{aligned}
$$

and we have that

$$
L_{0}=\frac{1}{4}|A \times B|^{2}+\frac{1}{4}|B \times C|^{2}+\frac{1}{4}|C \times A|^{2} .
$$


Corollary 1. If $A, B$ and $C$ are not collinear then the niveau surfaces of the function $\Sigma$ are convex and compact.

3. A special class of generalized conics with infinitely many focal points

Let $\gamma:[a, b] \rightarrow \mathbb{R}^{3}$ be a continuous, piecewise smooth curve under the partition $a=t_{0}<t_{1}<\ldots<t_{n-1}=t_{n}=b$ and consider the function

$$
P \rightarrow \mathcal{A}(P)
$$

measuring the area of generalized cones with such a curve as the common directrix and the vertex $P$.

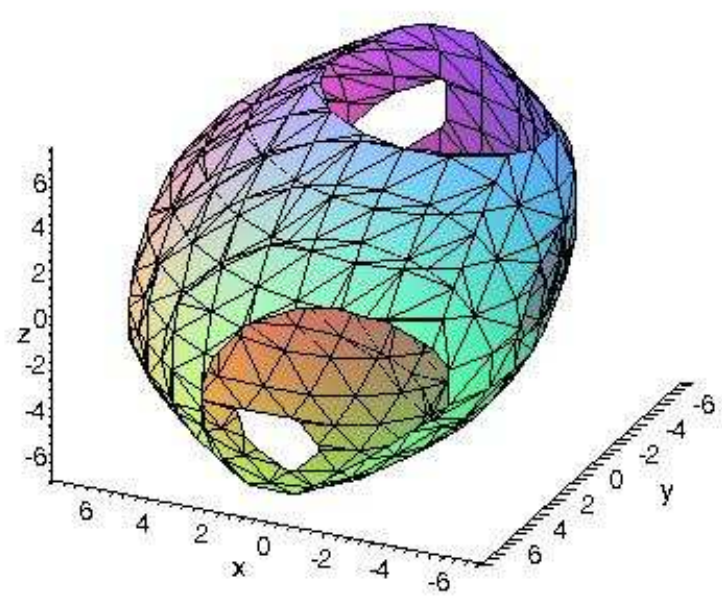

Figure 1. A niveau surface with $A=(1,0,0), B=(0,1,0)$ and $C=(0,0,1)$.

Definition 1. Niveau surfaces of the form $\mathcal{A}(P)=$ const. are called awnings spanned by $\gamma$.

Using the parameterization

$$
\vec{r}:[0,1] \times[a, b] \rightarrow \mathbb{R}^{3}, \quad \vec{r}(u, v):=u P+(1-u) \gamma(v)
$$




$$
\text { "vincze" — 2009/5/28 — 21:20 — page } 93 \text { — \#7 }
$$

an easy calculation shows that

$$
D_{1} \vec{r}(u, v)=P-\gamma(u), \quad D_{2} \vec{r}(u, v)=(1-u) \gamma^{\prime}(v)
$$

and, consequently,

$$
D_{1} \vec{r}(u, v) \times D_{2} \vec{r}(u, v)=(1-u)(P-\gamma(v)) \times \gamma^{\prime}(v)
$$

provided that $v$ is not one of the parameters $t_{i}^{\prime} s$. Then the area function can be calculated by the formula

$$
\mathcal{A}(P)=\sum_{i=1}^{n} \int_{t_{i-1}}^{t_{i}} \int_{0}^{1}\left|D_{1} \vec{r} \times D_{2} \vec{r}\right| d u d v .
$$

Particularly,

$$
\mathcal{A}(P)=\frac{1}{2} \sum_{i=1}^{n} \int_{t_{i-1}}^{t_{i}}\left|(P-\gamma) \times \gamma^{\prime}\right| d v .
$$

THEOREM 2. Any awning is the boundary of a generalized conic with the set of foci $G:=\{\vec{g} \mid \vec{g}=\gamma(v)$, where $v \in[a, b]\}$ and the weight function

$$
\alpha(\vec{g}, P):=\frac{1}{2} \sin (\text { the angle of } P-\vec{g} \text { and the tangent line of } G \text { at } \vec{g}) .
$$

Proof. Let $\vec{g}=\gamma(v)$ be a point in $G$. Then

$$
\frac{1}{2}\left|(P-\gamma(v)) \times \gamma^{\prime}(v)\right|=|P-\vec{g}|\left|\gamma^{\prime}(v)\right| \alpha(\vec{g}, P)=d(\vec{g}, P) \alpha(\vec{g}, P)\left|\gamma^{\prime}(v)\right| .
$$

Integrating by $v$ we have that

$$
\mathcal{A}(P)=\int_{G} d(\vec{g}, P) \alpha(\vec{g}, P) d g
$$

and the niveau surfaces of $\mathcal{A}$ are the boundary of generalized conics of the form

$$
\int_{G} d(\vec{g}, P) \alpha(\vec{g}, P) d g \leq \text { const. }
$$

as was to be stated.

Lemma 4. The function $P \rightarrow \mathcal{A}(P)$ is convex.

Proof. The proof is similar to that of Lemma 1 taking the affine term

$$
f(P)=P \times \gamma^{\prime}(v)-\gamma(v) \times \gamma^{\prime}(v)
$$

for some fixed parameter $v$. 


$$
\text { "vincze" — 2009/5/28 - 21:20 — page } 94-\# 8
$$

REMARK 3. If the curve $\gamma$ runs through the sides of the triangle $A B C$ then we get back to the basic geometric idea.

REMARK 4. Using the parameterization

$$
\vec{r}\left(u, v_{1}, \ldots, v_{n-2}\right)=u P+(1-u) \gamma\left(v_{1}, \ldots, v_{n-2}\right),
$$

where $\gamma: H \rightarrow \mathbb{R}^{n}$ is a parameterized surface of dimension $n-2$, the awnings can be given by

$$
\frac{1}{n-1} \int_{H}\left|(P-\gamma) \times D_{1} \gamma \times \ldots \times D_{n-2} \gamma\right| d v_{1} \ldots d v_{n-2}=\text { const. }
$$

in case of higher dimensional spaces.

\section{Awnings spanned by a circle}

Let $\gamma$ be a parameterized circle, i.e.

$$
\gamma(v):=(r \cos v, r \sin v, 0)
$$

where $0 \leq v \leq 2 \pi$. Awnings spanned by $\gamma$ can be given by the integral equation

$$
\frac{r}{2} \int_{0}^{2 \pi} \sqrt{z^{2}+(x \cos v+y \sin v-r)^{2}} d v=\text { const. }
$$

They are obviously invariant under the rotation around the $z$-axis. In what follows we investigate them in details.

Lemma 5. Awnings spanned by a circle are convex compact surfaces in the space. The global minimum of the function

$$
\mathcal{A}(P):=\frac{r}{2} \int_{0}^{2 \pi} \sqrt{z^{2}+(x \cos v+y \sin v-r)^{2}} d v, \quad P=P(x, y, z)
$$

is attained at any point contained by the convex hull of the circle.

Proof. Let $n \geq 3$ and consider the vertices $A_{0}, \ldots, A_{n}=A_{0}$ of the inscribed regular $n$-gon with a fixed initial point $A_{0}$ as the set of foci. It can be easily seen that

$$
\lim _{n \rightarrow \infty} \Sigma_{n}(P)=\mathcal{A}(P)
$$




$$
\text { "vincze" — 2009/5/28 — 21:20 — page } 95 \text { — \#9 }
$$

and the set of the extremal points is just the limit

$$
\lim _{n \rightarrow \infty} \operatorname{conv}\left\{A_{0}, \ldots, A_{n}\right\}
$$

of the convex hulls.

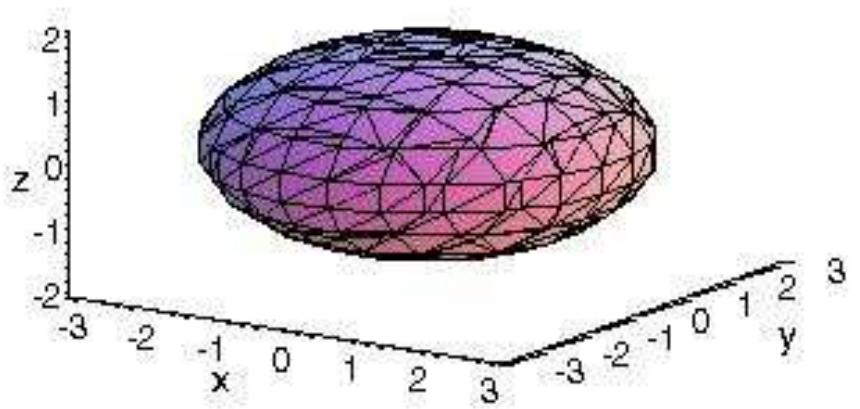

Figure 2. An awning spanned by the unit circle.

5. Approximation of awnings by quadrics

THEOREM 3. The volume enclosed by the awning

$$
\frac{r}{2} \int_{0}^{2 \pi} \sqrt{z^{2}+(x \cos v+y \sin v-r)^{2}} d v=c
$$

is greater than or equal to

$$
V_{1}:=\frac{8}{3} \pi\left(\frac{c^{2}}{r^{2} \pi^{2}}-r^{2}\right)^{\frac{3}{2}}
$$

Proof. Since

$$
\left(\int_{0}^{2 \pi} f d v\right)^{2} \leq 2 \pi \int_{0}^{2 \pi} f^{2} d v
$$




$$
\text { "vincze" — 2009/5/28 - 21:20 — page } 96 \text { — \#10 }
$$

we have that

$$
c^{2} \leq \frac{r^{2}}{2} \pi \int_{0}^{2 \pi} z^{2}+(x \cos v+y \sin v-r)^{2} d v .
$$

Therefore the surface defined by the equation

$$
c^{2}=\frac{r^{2}}{2} \pi \int_{0}^{2 \pi} z^{2}+(x \cos v+y \sin v-r)^{2} d v
$$

is contained by the convex hull of the awning. Here

$$
c^{2}=\frac{r^{2}}{2} \pi^{2}\left(x^{2}+y^{2}+2 r^{2}+2 z^{2}\right),
$$

which gives an ellipsoid

with

$$
1=\frac{x^{2}}{\alpha^{2}}+\frac{y^{2}}{\beta^{2}}+\frac{z^{2}}{\gamma^{2}}
$$

$$
\alpha^{2}=\beta^{2}=2\left(\frac{c^{2}}{r^{2} \pi^{2}}-r^{2}\right) \text { and } \gamma^{2}=\frac{c^{2}}{r^{2} \pi^{2}}-r^{2}
$$

Its volume is stated as a lower bound.

REMARK 5. The surfaces has a contact point of first order with

$$
x_{0}=y_{0}=0 \quad \text { and } \quad z_{0}= \pm\left(\frac{c^{2}}{r^{2} \pi^{2}}-r^{2}\right)^{\frac{1}{2}} .
$$

To find an upper bound for the volume enclosed by the awning we can use the vertices $A_{0}, \ldots, A_{n}=A_{0}$ of the inscribed regular $n$-gon with a fixed initial point $A_{0}$. According to the convexity, we have that

$$
\Sigma_{n}(P) \leq \mathcal{A}(P)
$$

and, consequently,

$$
\frac{1}{4} \sum_{i}\left|\left(P-A_{i}\right) \times\left(P-A_{i+1}\right)\right|^{2} \leq\left(\Sigma_{n}(P)\right)^{2} \leq c^{2} .
$$

Therefore the surface defined by the equation

$$
\frac{1}{4} \sum_{i}\left|\left(P-A_{i}\right) \times\left(P-A_{i+1}\right)\right|^{2}=c^{2}
$$


contains the awning in its convex hull. To simplify the calculation as far as possible consider the case $n=3$ with $r=\sqrt{\frac{2}{3}}$. Rotating in the euclidean sense if necessary we can suppose that

$$
A_{0}=(1,0,0), \quad A_{1}=(0,1,0) \quad \text { and } \quad A_{2}=(0,0,1)
$$

see Figure 1. Using the notations as above

$$
\frac{1}{4} \sum_{i}\left|\left(P-A_{i}\right) \times\left(P-A_{i+1}\right)\right|^{2}=L_{2}(x, y, z)+L_{1}(x, y, z)+L_{0} .
$$

By the help of a straightforward calculation

$$
\begin{gathered}
L_{2}(x, y, z)=x^{2}+y^{2}+z^{2}+\frac{1}{2}(x y+y z+z x) \\
L_{1}(x, y, z)=-(x+y+z) \quad \text { and } \quad L_{0}=\frac{3}{4} .
\end{gathered}
$$

Taking the matrix

$$
\left(\begin{array}{ccc}
1 & 1 / 4 & 1 / 4 \\
1 / 4 & 1 & 1 / 4 \\
1 / 4 & 1 / 4 & 1
\end{array}\right)
$$

of the quadratic form, the eigenvalues are $3 / 2$ and $3 / 4$ with a system of eigenvectors

$$
b_{1}=\frac{1}{\sqrt{3}}\left(\begin{array}{l}
1 \\
1 \\
1
\end{array}\right), \quad b_{2}=\frac{1}{\sqrt{2}}\left(\begin{array}{c}
-1 \\
0 \\
1
\end{array}\right) \quad \text { and } \quad b_{3}=\frac{1}{\sqrt{6}}\left(\begin{array}{c}
-1 \\
2 \\
-1
\end{array}\right)
$$

Using the coordinate transformation

$$
\left(\begin{array}{ccc}
\frac{1}{\sqrt{3}} & -\frac{1}{\sqrt{2}} & -\frac{1}{\sqrt{6}} \\
\frac{1}{\sqrt{3}} & 0 & \frac{2}{\sqrt{6}} \\
\frac{1}{\sqrt{3}} & \frac{1}{\sqrt{2}} & -\frac{1}{\sqrt{6}}
\end{array}\right)\left(\begin{array}{l}
\tilde{x} \\
\tilde{y} \\
\tilde{z}
\end{array}\right)=\left(\begin{array}{l}
x \\
y \\
z
\end{array}\right)
$$

it follows that

$$
L_{2}(\tilde{x}, \tilde{y}, \tilde{z})+L_{1}(\tilde{x}, \tilde{y}, \tilde{z})+L_{0}=\frac{3}{2} \tilde{x}^{2}+\frac{3}{4} \tilde{y}^{2}+\frac{3}{4} \tilde{z}^{2}-\sqrt{3} \tilde{x}+\frac{3}{4}
$$

and the left hand side of the equation

$$
L_{2}(x, y, z)+L_{1}(x, y, z)+L_{0}=c^{2}
$$




$$
\text { "vincze" — 2009/5/28 — 21:20 — page } 98 \text { — \#12 }
$$

can be written into the form

$$
\frac{\left(\tilde{x}-\tilde{x}_{0}\right)^{2}}{\alpha^{2}}+\frac{\tilde{y}^{2}}{\beta^{2}}+\frac{\tilde{z}^{2}}{\gamma^{2}}=1
$$

with

$$
\alpha^{2}=\frac{2 c^{2}}{3}-\frac{1}{6} \quad \text { and } \quad \beta^{2}=\gamma^{2}=2\left(\frac{2 c^{2}}{3}-\frac{1}{6}\right)
$$

THEOREM 4. The volume enclosed by the awning

$$
\frac{r}{2} \int_{0}^{2 \pi} \sqrt{z^{2}+(x \cos v+y \sin v-r)^{2}} d v=c, \quad r=\sqrt{\frac{2}{3}}
$$

is less than or equal to

$$
V_{2}:=\frac{8}{3} \pi\left(\frac{2 c^{2}}{3}-\frac{1}{6}\right)^{\frac{3}{2}}
$$

ThEOREM 5. The volume enclosed by the awning

$$
\Sigma_{3}(P)=c, \quad A_{0}=(1,0,0), \quad A_{1}=(0,1,0) \quad \text { and } \quad A_{2}=(0,0,1)
$$

is between

$$
V_{1}:=\frac{8}{3} \pi\left(\frac{2 c^{2}}{9}-\frac{1}{6}\right)^{\frac{3}{2}} \quad \text { and } \quad V_{2}:=\frac{8}{3} \pi\left(\frac{2 c^{2}}{3}-\frac{1}{6}\right)^{\frac{3}{2}}
$$

Proof. According to Theorem 4 it is enough to investigate the lower bound. The Cauchy-Schwarz inequality shows that

$$
c^{2}=\left(\Sigma_{3}(P)\right)^{2} \leq 3\left(L_{2}(x, y, z)+L_{1}(x, y, z)+L_{0}\right) .
$$

Therefore the surface defined by the equation

$$
c^{2}=3\left(L_{2}(x, y, z)+L_{1}(x, y, z)+L_{0}\right)
$$

is contained by the convex hull of the awning. Similar calculations as above give rise to the ellipsoid

$$
\frac{\left(\tilde{x}-\tilde{x}_{0}\right)^{2}}{\alpha^{2}}+\frac{\tilde{y}^{2}}{\beta^{2}}+\frac{\tilde{z}^{2}}{\gamma^{2}}=1
$$

with

$$
\alpha^{2}=\frac{2 c^{2}}{9}-\frac{1}{6} \quad \text { and } \quad \beta^{2}=\gamma^{2}=2\left(\frac{2 c^{2}}{9}-\frac{1}{6}\right) .
$$

Its volume is stated as a lower bound. 


$$
\text { "vincze" — 2009/5/28 - 21:20 — page 99 — \#13 }
$$

\section{References}

[1] J. M. Borwein and A. S. Lewis, Convex Analysis and Nonlinear Optimization, Springer-Verlag, New York, Inc, 2000.

[2] C. Groß and T.-K. Strempel, On Generalization of Conics and on a Generalization of the Fermat-Toricelli problem, Amer. Math. Monthly 105, no. 8 (1998), 732-743.

ÁBRIS NAGY, ZSOLT RÁBAI and CSABA VINCZE

INSTITUTE OF MATHEMATICS

UNIVERSITY OF DEBRECEN

4010 DEBRECEN, P.O. BOX 12,

HUNGARY

E-mail: abrish@freemail.hu

E-mail: rabxly@vipmail.hu

E-mail: csvincze@math.klte.hu

(Received January, 2008) 